Research Paper

\title{
Cholesterol Derivatives Based Charged Liposomes for Doxorubicin Delivery: Preparation, In Vitro and In Vivo Characterization
}

\author{
Yu Nie ${ }^{1}$, Li Ji1, Hong Ding², $\mathrm{Li} \mathrm{Xie}^{1}, \mathrm{Li} \mathrm{Li}^{1}$, Bin $\mathrm{He}^{1}$, Yao Wu¹, Zhongwei Gu${ }^{\circledR}$ \\ 1. National Engineering Research Center for Biomaterials, Sichuan University, Chengdu 610064, Sichuan, P. R. China. \\ 2. Department of Immunology, Herbert Wertheim College of Medicine, Florida International University, Miami, FL33199, USA.
}

$\triangle$ Corresponding author: Zhongwei Gu, Professor. National Engineering Research Center for Biomaterials, Sichuan University, No. 29, Wangjiang Road, Chengdu, Sichuan, 610064, P. R. China. Tel.: +86-28-85410336 Fax: +86-28-85410653 E-mail: zwgu@scu.edu.cn.

( ) Ivyspring International Publisher. This is an open-access article distributed under the terms of the Creative Commons License (http://creativecommons.org/ licenses/by-nc-nd/3.0/). Reproduction is permitted for personal, noncommercial use, provided that the article is in whole, unmodified, and properly cited.

Received: 2012.07.30; Accepted: 2012.09.28; Published: 2012.11.13

\begin{abstract}
Cholesterol plays a critical role in liposome composition. It has great impact on the behavior of liposome in vitro and in vivo. In order to verify the possible effects from cholesterol charge, surface shielding and chemical nature, two catalogs of liposomes with charged and PEGylated cholesterols were synthesized. Anionic liposomes (AL) and cationic liposomes $(C L)$ were prepared, with charges from hemisuccinate and lysine in cholesterol derivatives, respectively. Characteristics of different formulated liposomes were investigated after doxorubicin encapsulation, using neutral liposomes (NL) as control. Results showed that after PEGylation, AL and $\mathrm{CL}$ liposomes displayed prolonged retention release profile, while kept similar size distribution, encapsulation efficiency, low cytotoxicity and hemolysis comparing with NL. Confocal laser scanning microscopy and flow cytometry experiments confirmed the significantly higher cell uptake from $A L$ and $C L$ vesicles than the NL in mouse breast carcinoma and melanoma cells, human epithelial carcinoma and hepatoma cells. It was in accordance with our corresponding cellular mortality studies of DOX-loaded liposomes. The in vivo anti-tumor effect experiments from charged liposomes also presented much higher tumor inhibition effect $(70 \%$ vs $45 \%, p<0.05)$ than NL liposomes. This is the first time reporting anti-cancer effect from charged cholesterol liposome with/without PEGylation. It may give deeper understanding on the liposome formulation which is critical for liposome associated drug research and development.
\end{abstract}

Key words: Liposome, Cholesterol derivative, charged, PEGylation, image, Drug delivery.

\section{Introduction}

Liposomes have been widely used in the therapeutic drug delivery, including small molecular drugs, proteins, genes (DNA or RNA) and diagnostic contrast reagents.[1-6] For example, the doxorubicin encapsulated liposome (Doxil) has been approved by FDA for ovarian cancer and Kaposi's sarcoma treatment in United States over 10 years.[7]

Although liposome has been successfully applied in the clinic, further efforts on the component optimization are still a hot topic in the research for satisfying requirements from clinic.[8-13] Changes of the liposomes' characteristics, including size, composition, surface properties and charge, may attribute for the application needed. In these factors, charge is believed to be one of the key factors affecting cellular adhesion/uptake and drug delivery.[14, 15] Normally, liposomes with cationic lipids are prone to binding cells than liposomes with anionic lipids due to electrostatic interaction with negatively charged cell membrane (sialic acids and phospholipid head 
groups). This is the reason why cationic liposomes were frequently used to improve in vitro and in vivo efficacy for drug delivery.[9-11] However, once cationic liposomes were administrated into systemic circulation by intravenous injection, the effect from blood clearance and reticuloendothelial system cannot be ignored.[16] Meanwhile, it has been reported that negatively charged liposomes displayed faster and greater endocytosis than neutral liposomes in some cells.[17-20] To clarify this confusion, Dr. Dan applied Debye-Huckel limit of the Poisson-Boltzmann model to study the effect of liposome charge on cellular adhesion. Results showed that this liposome-cell interaction is determined not only from the lipids charge in the liposome, but also from lipids composition and cell types.[15]

PEGylated lipids can contribute longer circulation for liposome because of the "steric stabilization" effect. With the surface hydrophilic protective layer from PEG chain, PEGylated liposome showed characterizations of more stability, sustained release, prolonged blood circulation time and reduced mononuclear phagocyte system uptake. Although the supplementation of PEGylation into liposome can neutralize or reduce the net zeta potential of charged liposomes, the expected shielding effect in vivo was greatly diversified. From Dr. Torchlin's studies, liposomes containing phosphatidic acid or phosphatidyl serine had the similar size and charge. After PEGylation, it was found that only phosphatidic acid containing vesicles could extended circulation time, not phosphatidyl serine. There must be other factors we are not clear to affect these biological properties.[11]

Several studies have been reported that characterization of liposome composition, including charge and PEGylation, may have a marked influence on the in vitro and in vivo behavior of the carried chemotherapeutics. Besides phospholipids modification, cholesterol is well-known to work as the framework in liposomal membrane, which could reduce the fluidity stabilize the bilayer and control the drug permeability properties of liposomal membrane bilayer.[7] In the previous cholesterol studies, most of the researches were focused on the content effect; few studies were conducted on the cholesterol itself and its derivatives on the behavior of liposome so far, especially for the cholesterol charges. This critical effect factor from charged cholesterol and surface shielding on liposomal behavior are deserved to be explored.

Thus, in order to clarify this hypothesis, in this study, three types of cholesterol derivatives, including cholesterol hemisuccinate (CHEMS) with negative charge, lysine-based cholesterol (CHLYS) with posi- tive charge and PEGylated cholesterol (Chol-PEG) without charge were synthesized. These three types of cholesterols were incorporated with phospholipids to form liposome with according surface charge. Currently, imaging techniques or modalities have been incorporated into drug delivery system, especially in the nanomedicine field.[21-25] Comparing with traditional analysis, high resolution images can not only provide quick and accurate information of drug behaviors or new materials in the period of treatment, but also has close relationship with drug efficacy which can be called as theranostics. It can facilitate the localization and quantization of radioisotope or optical probe labeled materials in drug delivery study and clinic for early diagnosis and personal medicine.[26] In our study, with the assistance from fluorescent imaging and other classic assays, Doxorubicin (DOX), which has therapeutic effect with strong fluorescence to nucleus staining, was also designed to loaded into this three cholesterol sourced cationic, anionic and neutral liposomes with/without PEGylation to compare the behavior in vitro and in vivo.

\section{Materials and Methods}

\section{I. Materials}

L-a-phosphatidylcholine (egg hydrogenated, HEPC, 99\% deacetylated degree) was purchased from Q. P. Corporation Fine Chemical Division (Tokyo, Japan). Doxorubicin hydrochloride (DOX), cholesterol (Chol), methoxypoly(ethylene glycol) (mPEG, MW= $2000 \mathrm{Da}$ ) and dicyclohexylcarbodiimide (DCC) were purchased from Sigma-Aldrich Chemical Co. (USA). Boc-protected (Boc tert-butoxycarbonyl) lysine was purchased from Chengdu New Technology Company Limited (Chengdu, China). Three cholesterol derivatives (CHLYS, CHEMS and Chol-PEG) were synthesized in our group. The mouse breast carcinoma 4T1 cells, mouse melanoma B16F10 cells, human epithelial carcinoma Hela cells and human hepatoma HepG2 cells were purchased from Shanghai Institute of Biochemistry and Cell Biology (Chinese Academy of Sciences, Shanghai, China). 3-(4, 5-dimethylthiazol-2-yl)-2, 5-diphenyltetrazolium bromide (MTT) was purchased from Sigma (St. Louis, MO, USA). Cell culture media (RPMI 1640), fetal bovine serum (FBS), trypsin, and antibiotics were purchased from Gibco BRL (USA). All the other chemicals of analytical grade were purchased from local commercial suppliers.

\subsection{Synthesis of cholesterol derivatives}

\subsection{Synthesis of lysine-based cholesterol (CHLYS)}

Synthesis of CHLYS was performed according to 
our previous work with some modifications.[27] In brief, a solution of dicyclohexylcarbodiimide (DCC, $0.82 \mathrm{~g}, 4 \mathrm{mmol}$ ) in anhydrous dichloromethane $\left(\mathrm{CH}_{2} \mathrm{Cl}_{2}, 20 \mathrm{~mL}\right)$ was added dropwise to a mixed solution of cholesterol $(1.16 \mathrm{~g}, 3 \mathrm{mmol})$, Boc-protected lysine $(0.69 \mathrm{~g}, 2 \mathrm{mmol})$ and a few 4-dimethylaminopyridine (DMAP) in anhydrous $\mathrm{CH}_{2} \mathrm{Cl}_{2}(20 \mathrm{~mL})$. The mixed solution was stirred for 2 $\mathrm{h}$ at $0^{\circ} \mathrm{C}$ overnight at room temperature. The white precipitation of dicyclohexylurea (DCU) was removed by filtration. Obtained filtrate was condensed and purified by flash chromatography on silica gel $\left(\mathrm{CH}_{2} \mathrm{Cl}_{2} / \mathrm{MeOH}\right.$ 85:15, with trace TEA, $\left.\mathrm{R}_{\mathrm{f}}=0.15\right)$ to get CHLYS-Boc. Trifluoroacetic acid (1 mL) was dropwise added to a solution of CHLYS-Boc $(0.1 \mathrm{mmol})$ in anhydrous $\mathrm{CH}_{2} \mathrm{Cl}_{2}(1 \mathrm{~mL})$ at $0^{\circ} \mathrm{C}$ for Boc group detachment. After $2 \mathrm{~h}$ stirring at room temperature, product was concentrated and recrystallized in anhydrous diethyl ether, with yield of $0.36 \mathrm{~g}$ (71\%). IR $\mathrm{\gamma} / \mathrm{cm}^{-1}: 1738$ (ester, $\left.\mathrm{C}=\mathrm{O}\right) ;{ }^{1} \mathrm{HNMR}\left(\mathrm{CDCl}_{3}, \delta \mathrm{ppm}\right)$ : 0.68 (s, 3H, H-18), 0.91 (s, 3H, H-21), 2.29 (d, 2H, H-4), $3.11\left(\mathrm{~m}, 2 \mathrm{H},-\mathrm{CH}_{2}\right), 4.22\left(\mathrm{~m}, 1 \mathrm{H},-\mathrm{CH}_{2}-\mathrm{NH}\right), 4.65(\mathrm{~m}$, $1 \mathrm{H},-\mathrm{CH}), 5.08$ (d, 2H, $\left.-\mathrm{CH}_{2}-\mathrm{NH}\right), 5.38$ (t, 1H, H-6). (Supplementary Material: Figure S1 A)

\subsubsection{Synthesis of cholesterol hemisuccinate (CHEMS)}

Succinic anhydride ( $3.6 \mathrm{~g}, 36.0 \mathrm{mmol})$ was added to a solution of cholesterol $(5.0 \mathrm{~g}, 12.9 \mathrm{mmol})$ in anhydrous pyridine $(40 \mathrm{~mL})$. The reaction mixture was refluxed at $90{ }^{\circ} \mathrm{C}$ under nitrogen for $24 \mathrm{~h}$. The residue was washed with $\mathrm{HCl} /$ distilled ice water $(5: 95, \mathrm{v} / \mathrm{v})$. CHEMS can be obtained by recrystallized from acetone and dried under vacuum with yield of $5.3 \mathrm{~g}$, (85\%); mp 185 ${ }^{\circ} \mathrm{C}$. IR $\gamma / \mathrm{cm}^{-1}$ : 1734 (ester, $\mathrm{C}=\mathrm{O}$ ); ${ }^{1} \mathrm{H}$ NMR $\left(\mathrm{CDCl}_{3}, \delta\right.$ ppm): 0.67 (s, 3H, H-18), 0.91 (s, 3H, $\mathrm{H}-21), 2.32$ (d, 2H, H-4), $2.60\left(\mathrm{~m}, 2 \mathrm{H},-\mathrm{COCH}_{2}\right), 2.68$ $\left(\mathrm{m}, 2 \mathrm{H},-\mathrm{CH}_{2} \mathrm{CO}\right), 5.36(\mathrm{t}, 1 \mathrm{H}, \mathrm{H}-6)$. (Supplementary Material: Figure S1 B)

\subsubsection{Synthesis of CHEMS-PEG}

According to the protocol,[28] sulfonyl chloride $(0.67 \mathrm{~g}, 5 \mathrm{mmol})$ was added to a solution of CHEMS (2.4 g, $5 \mathrm{mmol}$ ) in $30 \mathrm{~mL}$ of anhydrous $\mathrm{CH}_{2} \mathrm{Cl}_{2}$ with $5 \mathrm{~h}$ refluxing under nitrogen stream. mPEG2000 (2 $\mathrm{g}$, 1 $\mathrm{mmol}$ ) was added and the reaction was continued for another $5 \mathrm{~h}$. At the end, the reaction mixture was condensed, and precipitated in diethyl ether thrice, recrystallized in anhydrous ethanol thrice and dried under vacuum. Dialysis was used to remove excessive mPEG (10 kDa cut-off), with yield of $2.2 \mathrm{~g}(88 \%)$. IR $\mathrm{\gamma} / \mathrm{cm}^{-1}$ : 1734 (ester, $\left.\mathrm{C}=\mathrm{O}\right)$; ${ }^{1} \mathrm{HNMR}\left(\mathrm{CDCl}_{3}, \delta \mathrm{ppm}\right)$ : 0.68 (s, 3H, H-18), 0.90 (s, 3H, H-21), 2.33 (d, 2H, H-4), $2.60\left(\mathrm{~m}, 2 \mathrm{H},-\mathrm{COCH}_{2}\right), 2.66\left(\mathrm{~m}, 2 \mathrm{H},-\mathrm{CH}_{2} \mathrm{CO}\right), 3.37$ (s, $\left.3 \mathrm{H},-\mathrm{CH}_{3}\right), 3.64\left(\mathrm{~m}, 176 \mathrm{H},-\mathrm{OCH}_{2} \mathrm{O}\right), 5.36(\mathrm{t}, 1 \mathrm{H}, \mathrm{H}-6)$.
(Supplementary Material: Figure S1 C)

\subsection{Preparation of various charged liposomes and drug loading}

Liposomes were prepared by traditional thin film-hydration method with different composition of HEPC, Chol, Chol-PEG and different charged components (CHLYS and CHEMS), respectively. Loading DOX into the liposomes was carried out by the remote loading method with an ammonium sulfate gradient.[29, 30] Briefly, different composition lipids were dissolved in $20 \mathrm{~mL}$ of chloroform/methanol (3:1, $\mathrm{v} / \mathrm{v}$ ), dried into thin film by rotary evaporator and hydrated in $10 \mathrm{~mL}$ of $120 \mathrm{mM}$ of ammonium sulfate solution. The mixture was further treated using a probe sonicator (UCX 130PB, sonics \& materials INC., USA) for $2 \mathrm{~min}$ at $50 \mathrm{~W}$. Unloaded ammonium sulfate was removed through dialysis against $0.9 \% \mathrm{NaCl}(10$ $\mathrm{kDa}$ cut-off). DOX solution was added to the liposomal dispersion with drug-to-lipid ratio of $1 / 10(\mathrm{w} / \mathrm{w})$ and incubated at $60^{\circ} \mathrm{C}$ for $2 \mathrm{~h}$. Commonly, the liposome formulations were stored at $4{ }^{\circ} \mathrm{C}$, and extruded through 0.22- micron filter for sterilization before use.

\subsection{Size distribution, morphology and zeta potential}

The particle size distribution and surface charge of liposomes $(0.5 \mathrm{mg} / \mathrm{mL})$ in phosphate-buffered saline (PBS, pH 7.4) were measured by dynamic laser-light scattering (DLS) by Zetasizer (Nano ZS, Malvern Instruments Ltd., UK). Liposomes were placed on copper grid films and stained with $2 \%$ $(\mathrm{w} / \mathrm{v})$ phosphotungstic acid for morphological observation by transmission electron microscopy (TEM) (JEM-100CX , JEOL, Japan), while dropped on silica slices for scanning electron microscopy (SEM) (Hitachi S4800, Japan) review. Surface properties of the liposomes were observed by atomic force microscopy (AFM) (MFP-3D, Asylum Research, USA) on mica slices.

\subsection{Drug entrapment efficiency}

Untrapped free DOX was separated from liposomes by size exclusion chromatography (SEC) using a Sephadex G-75 column (GE Healthcare, Uppsala, Sweden) with UV detection at $480 \mathrm{~nm}$. Liposomes were collected and dissolved in 10\% Triton X-100 for the content measurement of DOX in the liposomes by UV-vis spectrophotometry (Lambda 650, Perkin Elmer, USA). The loading/entrapment efficiency was calculated by

Entrapment efficiency $(\%)=($ encapsulated drug in liposomes / amount of total drug) $\times 100 \%$. 


\subsection{In vitro DOX release from liposomes}

Release studies were performed at $37^{\circ} \mathrm{C}$ in PBS buffer ( $\mathrm{pH}$ 7.4). Liposomes with a final DOX concentration of $0.5 \mathrm{mg} / \mathrm{mL}$ were added into a dialysis tube (10 kDa cut-off), and stirred in PBS medium at $37^{\circ} \mathrm{C}$ under perfect sink conditions. At predetermined time intervals, $1 \mathrm{~mL}$ of the medium was taken out for concentration detection by UV-vis spectrophotometry. Same volume of fresh media was fulfilled.

\subsection{Cytotoxicity study}

Cytotoxicity was determined by classic MTT assay. Briefly, 4T1 cells were seeded in 96-well plate at concentration of $5 \times 10^{3}$ cells/well in 1640 medium and cultured overnight. Drug-free liposome formulations with/without $5 \mathrm{~mol} \%$ Chol-PEG were added to reach a final concentration of $200 \mu \mathrm{g} / \mathrm{mL}$, and incubated for $24 \mathrm{~h}$. Measurement was performed in microplate reader (Model 550, Bio-Rad, USA).

\subsection{Hemolysis assay}

Hemolytic activity was evaluated by determining hemoglobin release from erythrocyte after incubation with different drug-free liposome formulations (cationic, anionic and neutral ones with/without 5 mol\% Chol-PEG). Briefly, rabbit blood samples were harvested from arteria auricularis into test tubes containing $124 \mathrm{mM}$ sodium citrate (sodium citrate: blood $=1: 9, \mathrm{v} / \mathrm{v}$ ), centrifuged and washed with saline. The obtained red blood cells (RBC, $1 \mathrm{~mL}$ ) were diluted with saline to $10 \mathrm{~mL}$. $0.5 \mathrm{~mL}$ of the RBC suspension was incubated with $2 \mathrm{~mL}$ of liposomes $(2.5 \mathrm{mg} / \mathrm{mL})$ at $37^{\circ} \mathrm{C}$ with gentle shaking. After $1 \mathrm{~h}$, the samples were centrifuged at $3000 \mathrm{rpm}$ for $5 \mathrm{~min}$. The absorbance (A) of the supernatant was measured by UV-vis spectrophotometry at $545 \mathrm{~nm}$. A negative control was prepared by mixing $0.5 \mathrm{~mL}$ of the $\mathrm{RBC}$ suspension with 2 $\mathrm{mL}$ of saline $(0 \%$ lysis), using water as a positive control $(100 \%$ lysis). The absorbance value of positive should be $0.8 \pm 0.3$, while negative one should be less than 0.03.[31] The hemolytic rates of the samples were calculated as the following equation (Eq. 1):

Hemolytic rate $(\%)=\left[\left(\mathrm{A}_{\mathrm{t}}-\mathrm{A}_{\mathrm{nc}}\right) /\left(\mathrm{A}_{\mathrm{pc}}-\mathrm{A}_{\mathrm{nc}}\right)\right] \times 100 \%$ ...(1)

where $A_{t}$ represents absorbance value of test sample, $A_{n c}$ and $A_{p c}$ stand for absorption value of negative and positive controls, respectively.

\subsection{Cellular uptake of DOX-loaded PEGylated liposomes}

Cellular uptake was examined by confocal laser scanning microscopy (CLSM, TCP SP5, Leica, Germany) and flow cytometry (Cytomics FC500, Beck- man Coulter).Various carcinoma cells including 4T1, B16F10, Hela and HepG2 were seeded on a $35 \times 12$ mm cell culture dish (NEST Biotech Co., LTD, China) for CLSM view, or on 6-well plate for flow cytometry measurement with cell population of $1 \times 10^{6}$ cells. Drug-loaded PEGylated liposomes were added and incubated for $0.5,1$ and $2 \mathrm{~h}$ at final DOX concentration of $5 \mu \mathrm{g} / \mathrm{mL}$. Cells were rinsed thrice with ice-cold PBS and observed by CLSM with excitation/emission at $488 / 590 \mathrm{~nm}$. In addition, cells were trypsinized and resuspended in $0.5 \mathrm{~mL}$ PBS buffer for fluorescence-activated cell sorting (FACS). At least $1 \times 10^{5}$ gated events per sample were collected.

\subsection{In vitro therapeutic test}

The cellular mortality study of DOX-loaded liposomes was performed against 4T1 cells on 96-well plate. Cells were exposed to different liposome formulations at a final DOX concentration of $5 \mu \mathrm{g} / \mathrm{mL}$ for $6,12,24,36$ and $48 \mathrm{~h}$, respectively. The viability was detected by MTT assay using DOX solution as control.

\section{I I. In vivo anti-tumor activity}

BALB/c mice (male, 18-22 g) were purchased from West China Experimental Animal Center of Sichuan University (China). They were maintained in a germ-free environment and allowed free access to food and water. All animal experiments were agreed to requirement from our IACUC and NIH guidelines for care and use of research animals. $5 \times 10^{5} 4 \mathrm{~T} 1$ cells in $50 \mu \mathrm{l}$ sterile PBS was embedded at the left flank of BALB/c mice by subcutaneous injection. After the volume of inoculated tumor reached approximately $100 \mathrm{~mm}^{3}$, mice were divided into 6 groups randomly (6 mice per group), and received different formulations along with controls via tail vein injection at a dose of $5 \mathrm{mg}$ DOX/ $\mathrm{kg}$ body weight on the $0,4 \mathrm{th}, 8 \mathrm{th}$ and 12th day, respectively. The transition of tumor volume and body weight was measured every three days thereafter. Tumor volume was calculated using Eq. (2) given below:

$$
V\left[\mathrm{~mm}^{3}\right]=1 / 2 \times L W^{2}
$$

Where, $L$ and $W$ stand for the lengths in long and short directions of the tumors, respectively. On the 21st days, all mice were sacrificed. Tumor inhibition rates were calculated according to Eq. (3) as follows:

Tumor Inhibition Rate (TIR, \%) $=\left[\left(W_{c^{-}} W_{t}\right) / W_{c}\right] \times$

$$
100 \% \quad \text {...(3) }
$$

Here $W_{c}$ referred to the average weight of tumor in control group; $W_{t}$ referred to average weight of tumor in treated group.

An autopsy was performed and specimens of 
heart were collected for microscopic examination. Samples were fixed in $4 \%$ neutral buffered formaldehyde and embedded in paraffin. $5 \mu \mathrm{m}$ thick sections were placed on polylysine-coated slides and stained with hematoxylin and eosin (H\&E). The stained slides were pictured under microscope (BX60, Olympus, Japan) at $200 \times$ magnifications.

\section{Results and discussion}

\section{I. Characterization of liposomes}

Non-PEGylated liposomes, as well as PEGylated anionic liposomes (AL), cationic liposomes (CL) and neutral liposomes (NL) were prepared with according characterization in size distribution and zeta potential (Table 1). After incorporating $5 \mathrm{~mol} \%$ of PEGylated component, all liposomes displayed narrower size distribution (all around $100 \mathrm{~nm}$ ) than conventional non-PEGylated ones. The parameters of phosphalipids sources, ratio and charge can only influence the average size in a limited level. The images of SEM,
AFM and TEM images (Figure 1A, B and C) showed that PEGylated AL liposomes (with or without DOX) was in spherical shape, and the encapsulation process did not affect the size and zeta potential. Similar results were obtained from DLS measurement (Table 1). Particles aggregation can be observed in non-PEGylated liposomes after one day's storage at $4^{\circ} \mathrm{C}$, which cannot be found for PEGylated liposomes.

As expected, zeta potential has close relationship with the charge on the cholesterol derivatives. Liposomes prepared from HEPC/Chol $(70 / 30, \mathrm{~mol} / \mathrm{mol})$ were neutral $(-2.0 \mathrm{mV})$. While adding $15 \mathrm{~mol} \%$ CHLYS or CHEMS, zeta potential changed to +37.2 $\mathrm{mV}$ or $-33.5 \mathrm{mV}$, respectively (Table 1). Introduction of PEG to charged liposomes reduced the absolute value of surface charges (from $+37.2 \mathrm{mV}$ to $+15.2 \mathrm{mV}$, or from $-33.5 \mathrm{mV}$ to $-14.6 \mathrm{mV}$, respectively). No significant change was found in the conventional neutral liposomes, which was consistent with previous research on the shielding effect of PEG.[32]

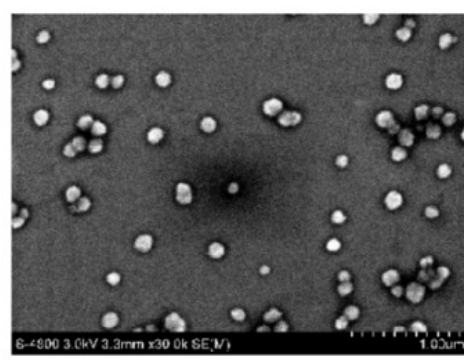

A

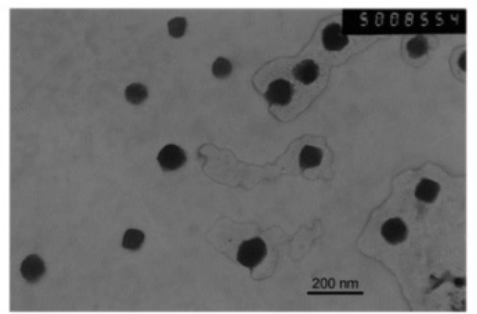

C
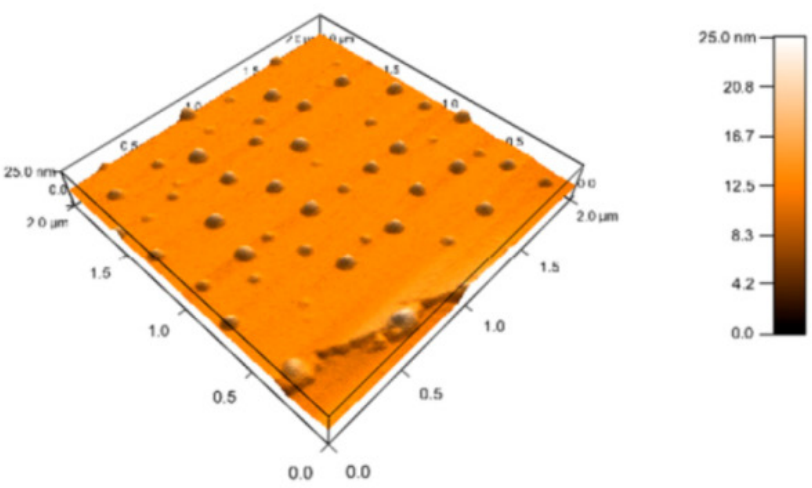

B

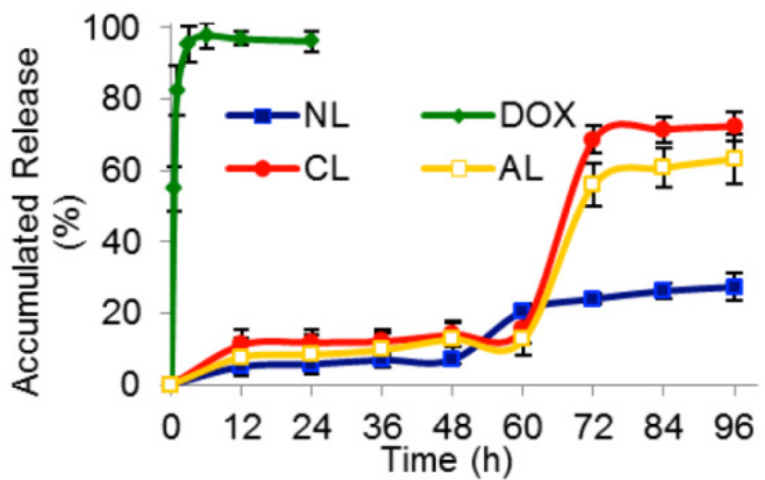

D

Figure I. The typical patterns of drug-free or DOX-loaded PEGylated anionic liposome (AL). (A) SEM image of drug-free AL. (B) Three dimensions AFM image of drug-free AL. (C) TEM image of DOX-loaded AL (bar= $200 \mathrm{~nm}$ ). (D) The DOX release profiles from different charged DOX-loaded PEGylated liposomes $(n=3)$. (NL: PEGylated neutral liposome, CL: PEGylated cationic liposome, AL: PEGylated anionic liposome, DOX: free DOX). 
Table I. Composition, size distribution and zeta potential of liposomes.

\begin{tabular}{lllll}
\hline Liposome type * $^{*}$ & Molar radio & Particle size $(\mathrm{nm})$ & Zeta potential $(\mathrm{mV})$ & Entrapment efficiency $(\%)$ \\
\hline HEPC/CHOL & $70: 30$ & $151.3 \pm 91.5^{\dagger}$ & $-2.0 \pm 4.4$ & $95.3 \pm 4.7$ \\
HEPC/CHOL/CHLYS & $70: 15: 15$ & $163.1 \pm 101.6^{\dagger}$ & $37.2 \pm 6.2$ & $96.7 \pm 4.5$ \\
HEPC/CHOL/CHEMS & $70: 15: 15$ & $144.7 \pm 95.1^{\dagger}$ & $-33.5 \pm 5.4$ & $95.8 \pm 5.0$ \\
HEPC/CHOL/CHEMS-PEG (NL) & $65: 30: 5$ & $108.2 \pm 34.2$ & $-2.3 \pm 4.4$ & $96.6 \pm 3.9$ \\
HEPC/CHOL/CHLYS/CHEMS-PEG (CL) & $65: 15: 15: 5$ & $104.5 \pm 40.1$ & $15.2 \pm 5.8$ & $97.3 \pm 4.8$ \\
HEPC/CHOL/CHEMS/CHEMS-PEG (AL) & $65: 15: 15: 5$ & $106.1 \pm 39.8$ & $-14.6 \pm 5.1$ & $98.2 \pm 3.1$
\end{tabular}

*NL: PEGylated neutral liposome, CL: PEGylated cationic liposome, and AL: PEGylated anionic liposome.

† The size distribution is abroad.

\subsection{Drug entrapment and in vitro DOX re- lease from liposomes}

It was demonstrated that remote loading method could efficiently encapsulate DOX into liposomes, in which ammonium sulfate gradient existed. The higher intra-liposomal concentration of $\left(\mathrm{NH}_{4}\right)_{2} \mathrm{SO}_{4}$ than that of the medium was used as the driving force for the influx of amphipathic weak bases, such as DOX.[29] In the presence of $\mathrm{SO}_{4}{ }^{2-}$, a gel-like precipitate of DOX could form and be trapped inside the liposomes. Size exclusion chromatography was used to separate free DOX from DOX-loaded liposome suspension. As shown in the UV elution profile (Supplementary Material: Figure S2), the small molecule of free DOX was eluted from 100 to $120 \mathrm{~mL}$, while liposomes with diameter of $100 \mathrm{~nm}$ were first eluted from 7.0 to $9.5 \mathrm{~mL}$. The experiments also showed that there were no significant differences in drug entrapment efficiency among the formulations (charged and neutral, with PEG or not). All the entrapment efficiency were as high as 95\% (drug/lipid=1:10, w/w) (Table 1), which means the surface charge and PEGylation had negligible impact on DOX loading efficiency. These results are consistent with previous findings.[30] Additionally, the PEGylated DOX-loaded liposomes can keep colloidal stable form for 3 months when stored at $4^{\circ} \mathrm{C}$ without any drug leakage.

Drug release profiles studies were also performed (Figure 1D). Compared with $4 \mathrm{~h}$ complete release of free DOX, the release profiles of PEGylated liposomes were obviously delayed post 48 th h due to encapsulation induced retaining effect of DOX. Interestingly, neutral liposomes (NL) showed a more rapid release at 48 th $\mathrm{h}$ time point, while the release in both cationic (CL) and anionic (AL) liposomes formulation started at 60 th $\mathrm{h}$ point, and reached $70 \sim 80 \%$ accumulated release at $72 \mathrm{nd} h$. The possible mechanism is that DOX entrapped by gradient ammonium sulfate may exhibit biphasic release, including slow dissolution of precipitate and membrane diffusion.[33] The drug release behavior was firstly governed by the slow precipitate dissolution rate inside liposomes, which led to the sustained release for a long period of time.[34] And then the dissolved drug could diffuse from the lipid membrane. During the test, precipitation in dialysis tube was observed in neutral vesicles from about 72nd $h$ (data not shown), resulting in the incomplete release of DOX. Nevertheless, charged liposomes (AL and $\mathrm{CL}$ ) displayed more stable for their mutual repulsive force in the suspension system. It was assumed that after 60 th $\mathrm{h}$, cationic or anionic lipid membrane might have some structure defects due to higher electrostatic interactions on membrane permeability,[35] thus accelerating membrane diffusion and leading to complete DOX release.

\subsection{Cytotoxicity study}

Various drug-free liposomes with/without PEGylation showed significant difference in cytotoxicity (Figure 2A). Without PEGylation, AL group presented much higher cellular viability (about $87 \%$ ) than that of CL group (around $60 \%, p<0.05$ ). While after PEGylation, all charged liposomes showed negligible toxicity on $4 \mathrm{~T} 1$ cells, remaining more than $90 \%$ viability. It is consistent with the previous test on cationic vesicles.[14]

\subsection{Hemolysis assay}

Hemolysis study was performed to investigate the potential toxicity after the intravenous injection of PEGylated AL, CL and NL in vivo. The leakage of hemoglobin was used to quantitatively compare the membrane-damaging properties of these liposomes. As shown in Figure 2B, the conventional non-PEGylated liposomes without PEG showed much higher extents of hemolysis rate than the PEGylated liposomes at $2 \mathrm{mg} / \mathrm{mL}$ concentration $(p<0.05)$. Without 5\% CHEMS-PEG, NL and AL induced mild hemolysis $(\sim 17 \%)$ while cationic liposomes showed $\sim 55 \%$ hemolysis rate due to the serious destroys to red blood cell. These results were consistent with the study from Lam's group on different charged micelles.[36] It was also supported by several previous investigations, which indicated that the primary amines (positive charge) on the surface of materi- 
als/particles may induce hemolysis.[37] On the contrary, the hemolysis rates in all PEGylated liposomes were less than $5 \%$. It is known that, PEG is a highly hydrated polymer and has a high degree of segmental flexibility in aqueous solution. Thus, PEGylation is commonly considered to reduce the serious cellular interaction, [38, 39] and consequently reduce the damage to red blood cell. With the improved the biocompatibility, the PEGylated liposomes could be further explored for anti-tumor activity, cellular uptake and in vivo effect.

\subsection{Cellular uptake of DOX-loaded PEGylated liposomes}

Cellular uptake of liposomes was studied via confocal microscopy (Figure 3A) and flow cytometry analysis (Figure 3B). DOX showed the fastest cellular uptake and could be used for cell nucleus model because of its property of fluorescent and therapy. Figure $3 \mathrm{~A}$ showed AL treated cells presented approximately the same fluorescence intensity as cells in CL group on $4 \mathrm{~T} 1$ cells, which was much higher than that of NL at $2 \mathrm{~h}$ post-treatment. In addition, AL treated cells also showed the highest fluorescence intensity in all liposome formulations in Hela, HepG2 and B16 cells.

There were no obvious effects from the original size, morphology, cytotoxicity, encapsulation, release and hemolysis for PEGylated cationic and anionic liposomes, thus, the good cellular uptake of CL was likely determined by the positive charges that could induce electrostatic interaction with negatively charged cell membrane as previously reported.[40] Whereas observations with AL might be explained by Dan's research,[15] who found cell-liposome adhesion depends not only on liposome charge, but also on the cell type and specific endocytosis. Only when the

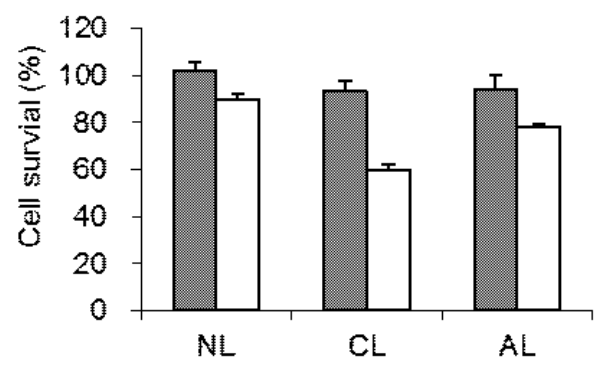

A cell-to-liposome charge ratio (either positive or negative) reaches the appropriate range for a specific cell type would facilitate the liposome uptake.[17] These phenomena were in accordance with previous research that negatively charged nanoparticles exhibited greater attraction to the macrophage and some tumor cells.[36, 41, 42] It might be due to distinct cell surface properties and specific endocytosis, since specific protein corona will form on the surface of liposomes after incubation with the cell medium.[43] Moreover, the quick release of DOX in the formulation with CHEMS might also contribute to the higher fluorescence intensity in AL group.[44] The detailed mechanisms involved are unclear, which required further investigation. The FACS data displayed the similar results and they have high consistent with results from confocal images (Figure 3B).

\subsection{In vitro cellular mortality}

In vitro anti-tumor activities of DOX-loaded PEGylated liposomes were evaluated via the determination of cell mortality on 4T1 tumor cells at 6th, 12th, 24th, 36th and 48th h time point (Figure 4). Free DOX showed the highest cellular mortality at each time point, charged liposomes (CL and AL) showed slightly lower anti-tumor proliferation, and the rate of NL was the lowest one among all formulations. For example, at 24th h of incubation time point, cell viability was about $30 \%$ for free DOX, AL and CL had approximately $43 \%$ of survived cells, and NL-treated group showed the highest cell viability of about $56 \%$ $(p<0.05)$. This was in accordance with cellular uptake experiment that charged liposomes had lower entrance than free DOX, but a greater extent of endocytosis than neutral ones.

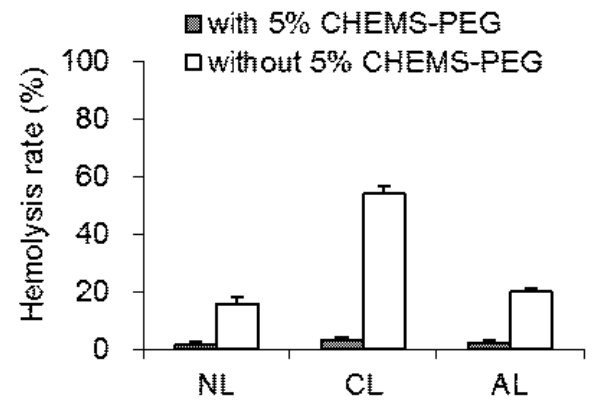

$\mathrm{B}$

Figure 2. Cytotoxicity of drug-free liposome with or without 5 mol\% Chol-PEG $(n=3)$. (A) Cell viability of $4 T$ I cells after incubation with drug-free liposome suspension $(200 \mu \mathrm{g} / \mathrm{mL}$ ). (B) Hemolysis rate of DOX-loaded liposome suspension $(2 \mathrm{mg} / \mathrm{mL})$ (NL: PEGylated neutral liposome, CL: PEGylated cationic liposome and AL: PEGylated anionic liposome). 
4T1
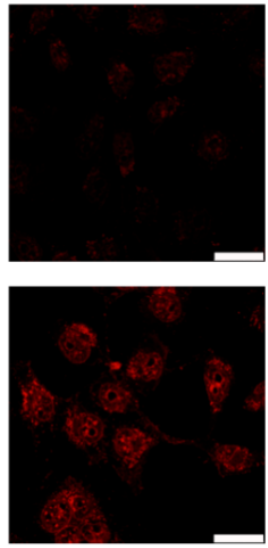

CL

AL
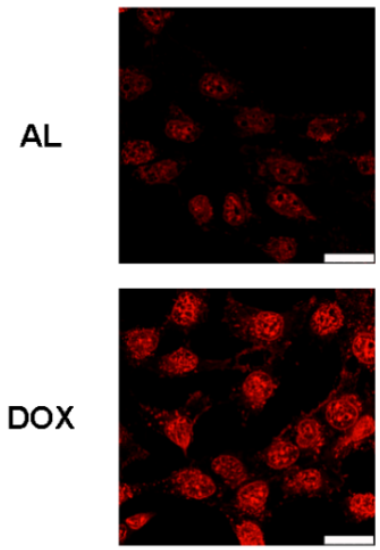

4T1
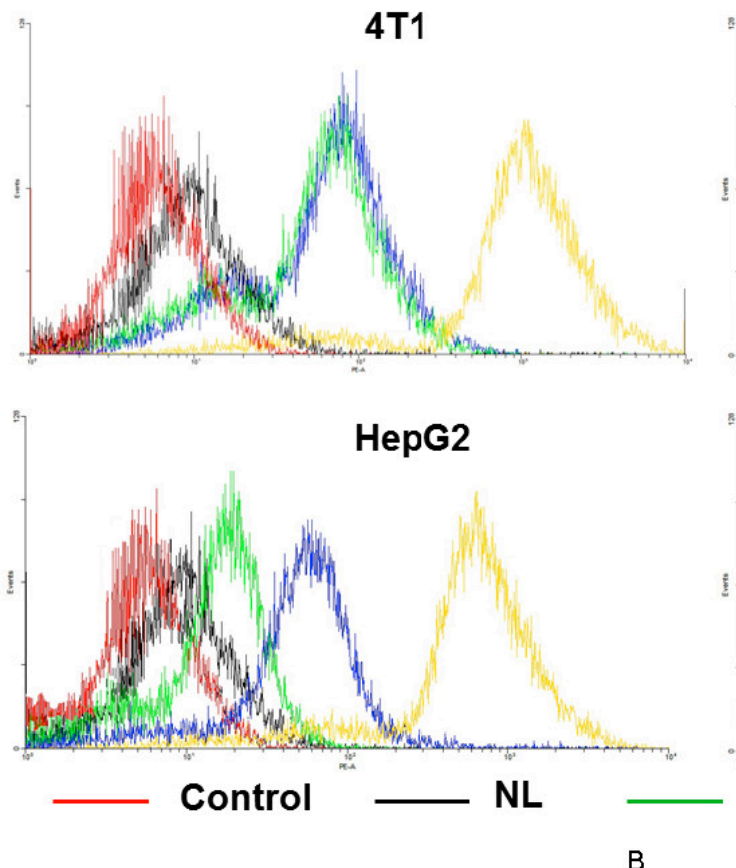

Hela
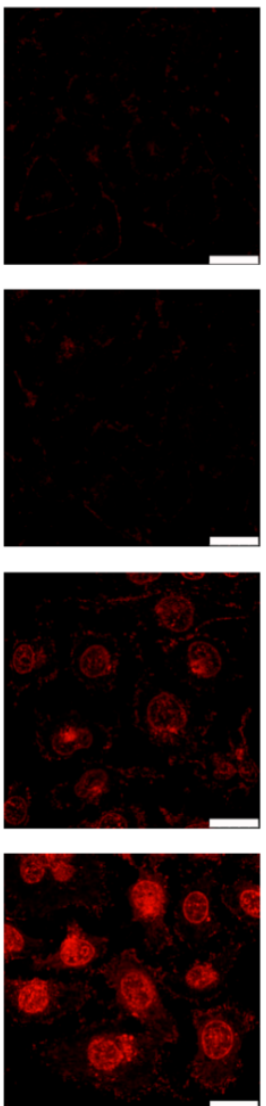

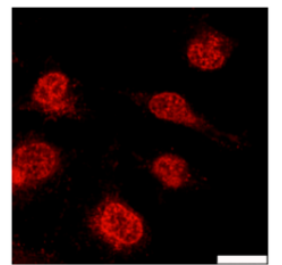

A
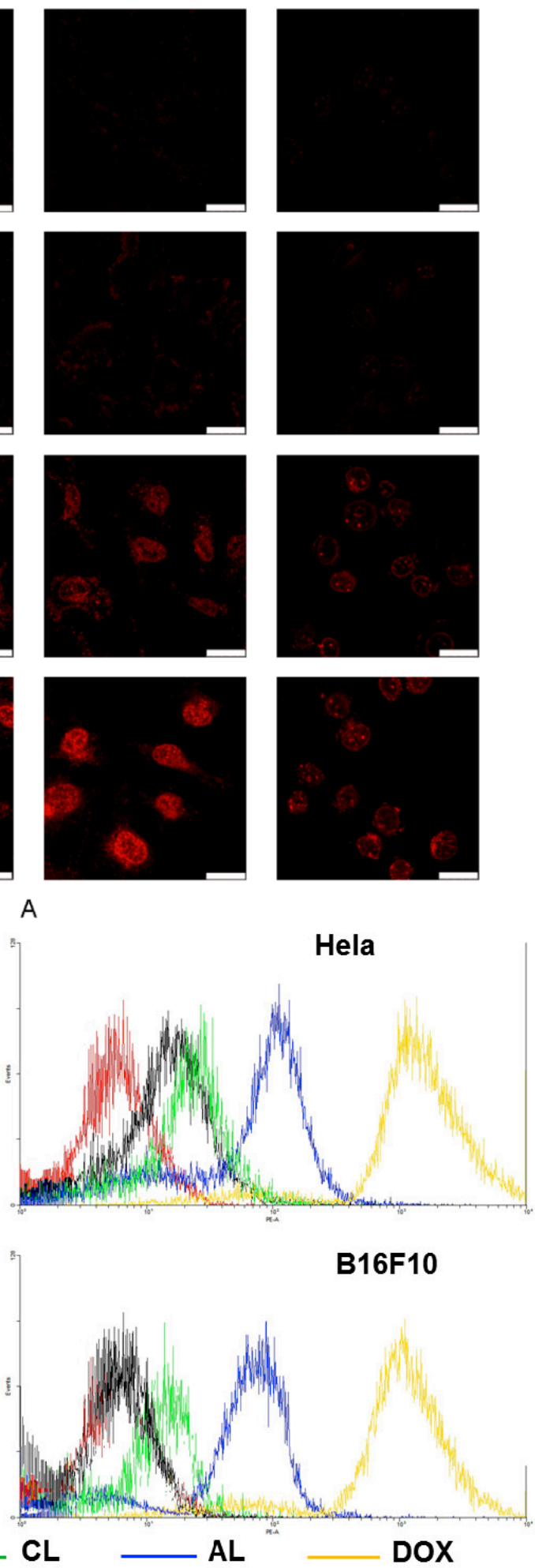
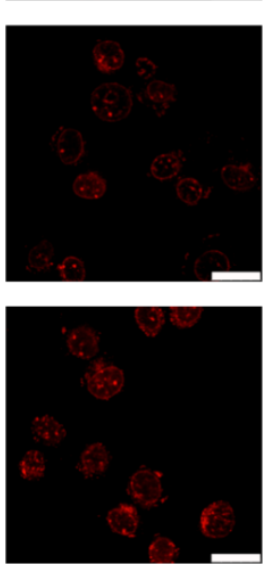

B16F10
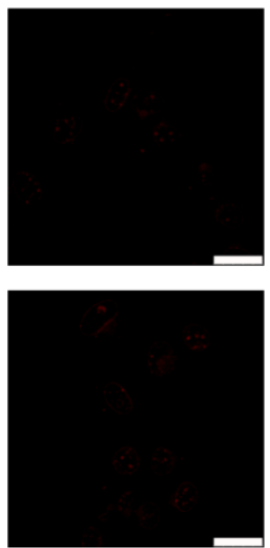

Hela

B16F10

DOX

Figure 3. Confocal microscopic images and flow cytometry results of various cells (4TI, Hela, HepG2 and BI6FI0) after incubation with free DOX or DOX-loaded PEGylated liposomes at $37^{\circ} \mathrm{C}$ for $2 \mathrm{~h}$. (A) Confocal microscopic images (All the images were taken at the same fluorescence intensity. bar $=25 \mu \mathrm{m}$ ) (B) Flow cytometry results. (Excitation: $488 \mathrm{~nm}$; emission: $590 \mathrm{~nm}$ ). 


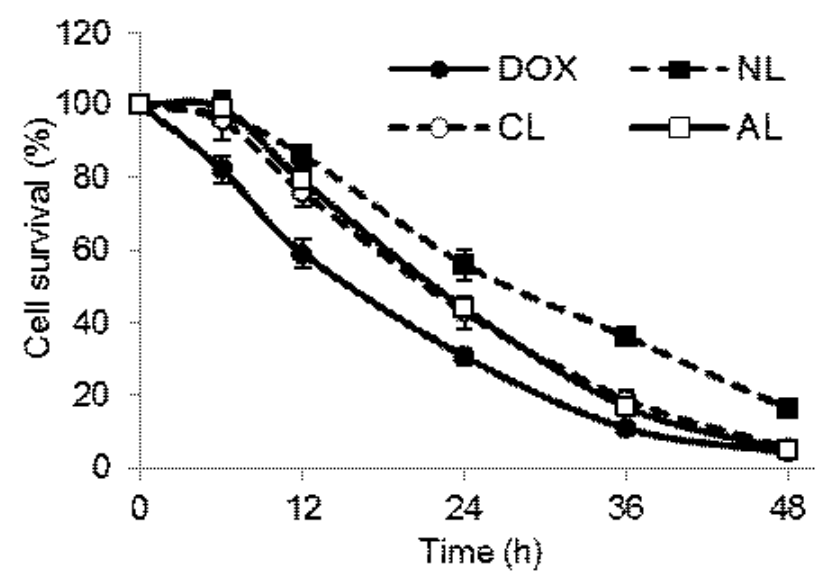

Figure. 4. Cell viability of 4TI cells after incubation with free DOX or DOX-loaded PEGylated liposomes at a final DOX concentration of 5 $\mu \mathrm{g} / \mathrm{mL}$ in various incubation time $(\mathrm{n}=5)$. (NL: PEGylated neutral liposome, $\mathrm{CL}$ : PEGylated cationic liposome and AL: PEGylated anionic liposome).

\subsection{In vivo anti-tumor activity}

In vivo anti-tumor activities of DOX-loaded PEGylated liposomes were tested using 4T1 breast cancer tumor-bearing BALB/c mice at a dose of $5 \mathrm{mg}$ $\mathrm{DOX} / \mathrm{kg}$ body weight. The change in the tumor volume was measured continuously during the experiment (Figure 5A) and the tumor inhibition rates were compared by tumor weight after all mice were sacrificed (Figure 5B). The direct observation of tumors excised from 4T1-bearing mice was also considered for evaluation. For the ease of graphical representation, representative three tumors were shown in Figure 5C. CL and AL treated groups showed high tumor growth suppression with $70.9 \%$ and $68.2 \%\left({ }^{* *} p\right.$ $>0.05$, CL group vs AL group), respectively. Meanwhile NL and free DOX displayed less tumor inhibitory effect with inhibition rate of $46.2 \%$ and $41.0 \%$, respectively. Different from the inhibition results in vitro, DOX encapsulated in AL and CL showed improved effect than free DOX in vivo ${ }^{*} p<0.05$ vs DOX group).

Although free DOX showed good cellular uptake, it was difficult to reach the tumor target site due to the rapid clearance by opsonization and uptake by the reticuloendothelial system.[45] Whereas DOX loaded liposomes could be delivered to the tumor site via enhanced permeability and retention effect. It was reported that cationic liposomes could induce significant tumor growth suppression than neutral vesicles and free drug, for preferential tumor accumulation.[9] In our study, the anti-tumor effect of PEGylated anionic liposomes was comparable to cationic ones in vivo. It might cause by the tumor cell type or the specific structure of CHEMS. In 4T1 cells, CL and AL showed similar uptake, indicating 4T1 cells prefer both cationic and anionic liposomes. As reported by
Hafez et al.,[35] CHEMS exhibited $\mathrm{pH}$ sensitive polymorphism, that when $\mathrm{pH}$ decreased to 5.5, CHEMS induced changes in the bilayer forming cylinder of liposome to the inverted hexagonal phase, thus accelerating drug release.

Significant toxicity was observed in the group only administrated with DOX. In the DOX group, $20 \%$ of body weight was found to lose in mice and displayed obvious no vigorousness in mice comparing with controls after the 3rd and 4th i.v. injection, which reflected the signs of systemic toxicity from DOX (Supplementary Material: Figure S3, ${ }^{*} p<0.05$ vs control and other liposome group). While mice treated with DOX-loaded liposomes showed none of these adverse effects $(p>0.05)$, indicating the low acute toxicity from the liposome formulation.

Considering the reported damage by free DOX to heart, such as swollen cardiac muscle fibers, interstitial edema and inflammatory infiltration,[46-48] histopathological investigation was conducted (Figure 5D). From the pathological data, no apparent changes were observed in drug-free or DOX-loaded liposome treatment groups. Thus, DOX-loaded PEGylated liposomes showed less cardiac toxicity in vivo as compared to free DOX.

Nowadays, research into the rational delivery and targeting of pharmaceutical, therapeutic, and diagnostic agents is at the forefront of projects in nanomedicine. These diagnostics based nanoparticles can improve the sensitivity and specificity and help identify abnormalities that cannot be detected with traditional ways, provide more effective and/ or more convenient routes of administration, lower therapeutic toxicity, extend the product life cycle, and ultimately reduce health-care costs. In the past few years, a number of nanoparticle-based therapeutic and diagnostic agents have been developed for the treat- 
ment of cancer, diabetes, pain, asthma, allergy, and infections. As a complicated therapeutic delivery system, liposome is also one of the most successful nanoparticles in the market approved by FDA for the chemotherapeutic drug DOX loading and delivery. Liposome allows passive targeted delivery and controlled release. With the assistance from diagnostic reagent or the fluorescence form the capsulated drug itself, liposome may be entity for drug delivery and medical imaging, which fit the property of nanomedicine with diagnostic functionality referred by the National Institutes of Health (NIH). The in vivo behavior of liposomes can be influenced by multiple interdependent factors. A minor change in chemical formulation may result in evident diversification in biological behavior.[33] To elucidate major factors that affect anti-tumor activity of $\mathrm{CL}$ and $\mathrm{AL}$, further in vivo biodistribution behavior including tumor localization and elimination need to be proceeded, which means the studies on physiological based pharmacokinetic (PBPK). DOX-loaded PEGylated charged liposomes exhibited strong anti-tumor efficiency both in vitro and in vivo, indicating that using cholesterol derivatives might be a promising approach in forming multi-functional liposomes for clinic.

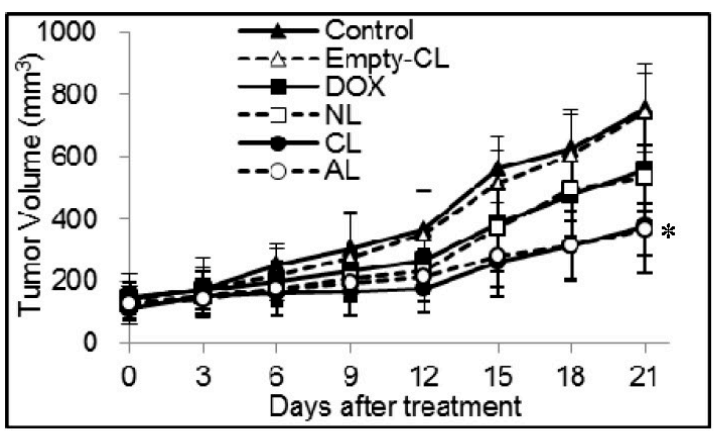

A

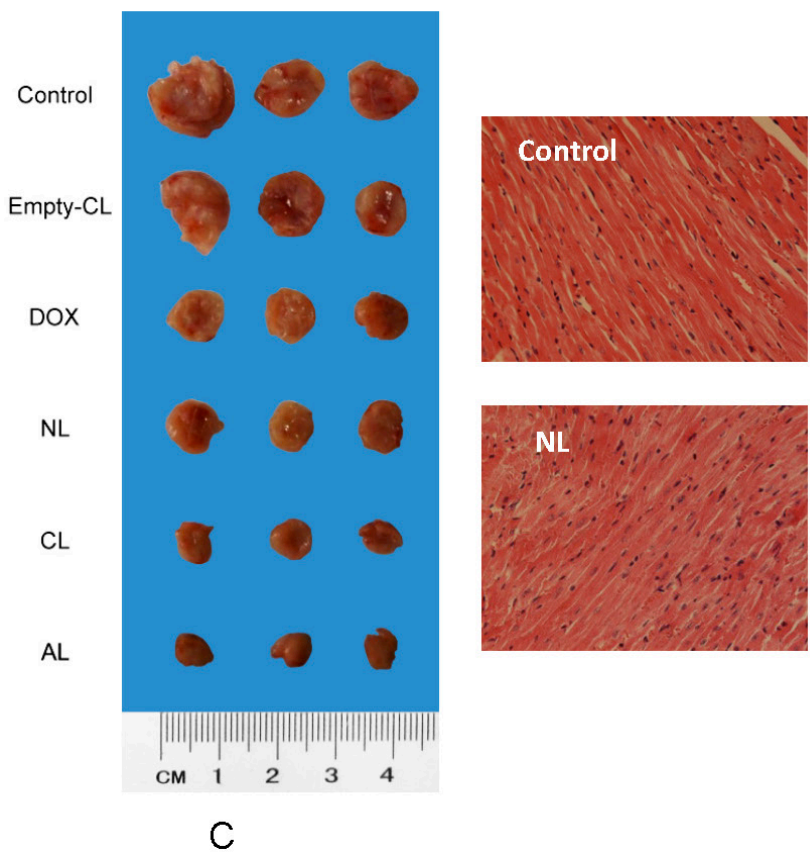

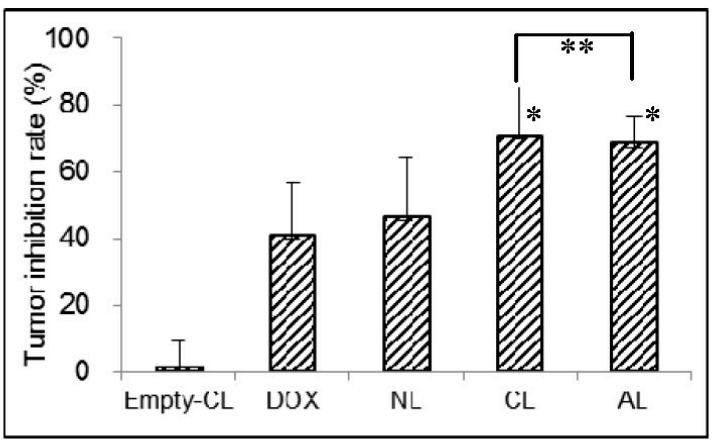

B
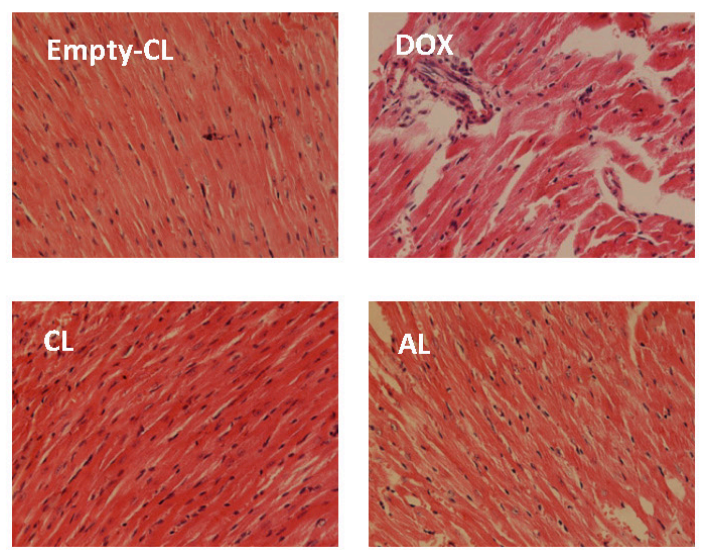

D

Figure 5. In vivo anti-tumor activity of liposomes after i.v. injection of different DOX-loaded PEGylated liposomes with a DOX concentration of $5 \mathrm{mg} / \mathrm{kg}$ body weight $(n=6)$. (A) The tumor volume in different treatment groups. ${ }^{*} p<0.05$ vs control group) (B) Tumor inhibition rates of various formulations. (*p<0.05 vs DOX group, ** $p>0.05$ ) (C) The tumor morphology after three weeks treatment of different DOX preparation. (D) Mouse myocardium: treated with free DOX or DOX-loaded liposomes at a DOX concentration of $5 \mathrm{mg} / \mathrm{kg}$ body weight (H\&E stain 200x). (NL: PEGylated neutral liposome, CL: PEGylated cationic liposome and AL: PEGylated anionic liposome). 


\section{Conclusion}

In our work, doxorubicin with property of fluorescence and therapy was successfully encapsulated into charged liposome formulations from cationic and anionic cholesterol derivatives. Such liposome formulations can serve as a model platform to further study the in vitro and in vivo behavior with assistance from fluorescent images, FACS technique and some other classic methods. Our research demonstrated that the charged liposomes incorporating with PEG showed prolonged and improved release profile of DOX, as well as low cytotoxicity and hemolysis. In addition, charged liposomes, especially the anionic ones have significant enhancement on uptake and cell inhibition in various carcinoma cells than neutral ones. In vivo tumor inhibition further confirmed that charged liposomes had greater tumor inhibitory effect, indicating both positively and negatively charged PEGylated liposome formulations with modified cholesterol derivatives may be potential drug carriers to improve the therapeutic efficacy.

\section{Supplementary Material}

Fig.S1 - S3. http://www.thno.org/v02p1092s1.pdf

\section{Acknowledgements}

The authors thank Drs. Gang Cheng and Yiyan He for their helpful discussions and suggestions, as well as Mr. Ming Li, Shuang Chang and Ms. Mingming Sheng for their technical assistance in sample preparation and analysis. This research was performed with support from the National Basic Research Program of China (National 973 program, No. 2011CB606206), National Science Foundation of China (NSFC, No. 81000657, 31271020, 51133004, 50830105) and Research Fund for the Doctoral Program of Higher Education of China (Grant No. 20100181120075), International S \& T Cooperation Program of Ministry of Science and Technology (No. 2010DFA51550), International Cooperation Project of Sichuan Province (2009HH0001)

\section{Competing Interests}

The authors have declared that no competing interest exists.

\section{References}

1. Lukyanov AN, Elbayoumi TA, Chakilam AR, Torchilin VP. Tumor-targeted liposomes: doxorubicin-loaded long-circulating liposomes modified with anti-cancer antibody. Journal of Controlled Release 2004; 100: 135-144.

2. Saad M, Garbuzenko OB, Minko T. Co-delivery of siRNA and an anticancer drug for treatment of multidrug-resistant cancer. Nanomedicine 2008; 3: 761-776.
3. Torchilin VP, Levchenko TS, Rammohan R et al. Cell transfection in vitro and in vivo with nontoxic TAT peptide-liposome-DNA complexes. Proc Natl Acad Sci U S A 2003; 100: 1972-1977.

4. Buyens K, De Smedt SC, Braeckmans K et al. Liposome based systems for systemic siRNA delivery: stability in blood sets the requirements for optimal carrier design. J Control Release 2012; 158: 362-370.

5. Park K. Comparative study on liposome targeting to tumor endothelium. J Control Release 2012; 158: 181.

6. Peng A, Kosloski M, Nakamura G et al. PEGylation of a Factor VIII-Phosphatidylinositol Complex: Pharmacokinetics and Immunogenicity in Hemophilia A Mice. The AAPS Journal 2012; 14: 35-42.

7. Addeo R, Faiola V, Guarrasi R et al. Liposomal pegylated doxorubicin plus vinorelbine combination as first-line chemotherapy for metastatic breast cancer in elderly women $>$ or $=65$ years of age. Cancer Chemother Pharmacol 2008; 62: 285-292.

8. Kuang Y, Liu J, Liu Z, Zhuo R. Cholesterol-based anionic long-circulating cisplatin liposomes with reduced renal toxicity. Biomaterials 2012; 33: 1596-1606.

9. Abu Lila AS, Kizuki S, Doi $\mathrm{Y}$ et al. Oxaliplatin encapsulated in PEG-coated cationic liposomes induces significant tumor growth suppression via a dual-targeting approach in a murine solid tumor model. J Control Release 2009; 137: 8-14.

10. Dragicevic-Curic N, Grafe S, Gitter B et al. Surface charged temoporfin-loaded flexible vesicles: in vitro skin penetration studies and stability. Int J Pharm 2010; 384: 100-108.

11. Wu J, Lee A, Lu Y, Lee RJ. Vascular targeting of doxorubicin using cationic liposomes. Int J Pharm 2007; 337: 329-335.

12. Levchenko TS, Rammohan R, Lukyanov AN et al. Liposome clearance in mice: the effect of a separate and combined presence of surface charge and polymer coating. Int J Pharm 2002; 240: 95-102.

13. Luk BT, Fang RH, Zhang L. Lipid- and Polymer-Based Nanostructures for Cancer Theranostics. Theranostics 2012.

14. Soenen SJ, Brisson AR, De Cuyper M. Addressing the problem of cationic lipid-mediated toxicity: the magnetoliposome model. Biomaterials 2009; 30: 3691-3701.

15. Dan N. Effect of liposome charge and PEG polymer layer thickness on cell-liposome electrostatic interactions. Biochim Biophys Acta 2002; 1564: 343-348.

16. Moghimi SM, Patel HM. Modulation of murine liver macrophage clearance of liposomes by diethylstilbestrol. The effect of vesicle surface charge and a role for the complement receptor Mac-1 (CD11b/CD18) of newly recruited macrophages in liposome recognition. J Control Release 2002; 78: 55-65.

17. Miller CR, Bondurant B, McLean SD et al. Liposome-cell interactions in vitro: effect of liposome surface charge on the binding and endocytosis of conventional and sterically stabilized liposomes. Biochemistry 1998; 37: 12875-12883.

18. Lee KD, Nir S, Papahadjopoulos D. Quantitative analysis of liposome-cell interactions in vitro: rate constants of binding and endocytosis with suspension and adherent J774 cells and human monocytes. Biochemistry 1993; 32: 889-899.

19. Allen TM, Austin GA, Chonn A et al. Uptake of liposomes by cultured mouse bone marrow macrophages: influence of liposome composition and size. Biochim Biophys Acta 1991; 1061: 56-64.

20. Lee KD, Hong K, Papahadjopoulos D. Recognition of liposomes by cells: in vitro binding and endocytosis mediated by specific lipid headgroups and surface charge density. Biochim Biophys Acta 1992; 1103: 185-197.

21. Wu F, Bhansali SG, Tamhane $\mathrm{M}$ et al. Noninvasive real-time fluorescence imaging of the lymphatic uptake of BSA-IRDye 680 conjugate administered subcutaneously in mice. J Pharm Sci 2012; 101: 1744-1754.

22. Ding H, Yong K-T, Roy I et al. Gold Nanorods Coated with Multilayer Polyelectrolyte as Contrast Agents for Multimodal Imaging. The Journal of Physical Chemistry C 2007; 111: 12552-12557.

23. Ding $\mathrm{H}, \mathrm{Wu}$ F. Images Guided Biodistribution and Pharmacokinetic Studies of Theranostics. Theranostics 2012; doi:10.7150/thno.4652.

24. Mura S, Couvreur P. Nanotheranostics for personalized medicine. Adv Drug Deliv Rev 2012.

25. Zhou Y, Chakraborty S, Liu S. Radiolabeled Cyclic RGD Peptides as Radiotracers for Imaging Tumors and Thrombosis by SPECT. Theranostics 2011; 1: 58-82.

26. Heneweer C, Gendy SE, Penate-Medina O. Liposomes and inorganic nanoparticles for drug delivery and cancer imaging. Ther Deliv 2012; 3: 645-656.

27. Li L, Nie Y, Zhu R et al. Preparation and gene delivery of alkaline amino acids-based cationic liposomes. Arch Pharm Res 2008; 31: 924-931. 
28. $\mathrm{Xu} \mathrm{H}$, Deng $\mathrm{Y}$, Chen $\mathrm{D}$ et al. Esterase-catalyzed dePEGylation of $\mathrm{pH}$-sensitive vesicles modified with cleavable PEG-lipid derivatives. J Control Release 2008; 130: 238-245.

29. Bolotin EM, Cohen R, Bar LK et al. Ammonium Sulfate Gradients for Efficient and Stable Remote Loading of Amphipathic Weak Bases into Liposomes and Ligandoliposomes. Journal of Liposome Research 1994; 4: 455-479.

30. Haran G, Cohen R, Bar LK, Barenholz Y. Transmembrane ammonium sulfate gradients in liposomes produce efficient and stable entrapment of amphipathic weak bases. Biochim Biophys Acta 1993; 1151: 201-215.

31. Duan $Y$, Nie $Y$, Gong $T$ et al. Evaluation of blood compatibility of MeO-PEG-poly (D,L-lactic-co-glycolic acid)-PEG-OMe triblock copolymer. Journal of Applied Polymer Science 2006; 100: 1019-1023.

32. Dadashzadeh S, Mirahmadi N, Babaei MH, Vali AM. Peritoneal retention of liposomes: Effects of lipid composition, PEG coating and liposome charge. J Control Release 2010; 148: 177-186.

33. Cui J, Li C, Guo W et al. Direct comparison of two pegylated liposomal doxorubicin formulations: Is AUC predictive for toxicity and efficacy? Journal of Controlled Release 2007; 118: 204-215.

34. Gardikis K, Tsimplouli C, Dimas K et al. New chimeric advanced Drug Delivery nano Systems (chi-aDDnSs) as doxorubicin carriers. Int J Pharm 2010; 402: 231-237.

35. Liu J, Jiang X, Ashley C, Brinker CJ. Electrostatically mediated liposome fusion and lipid exchange with a nanoparticle-supported bilayer for control of surface charge, drug containment, and delivery. J Am Chem Soc 2009; 131: 7567-7569.

36. Xiao K, Li Y, Luo J et al. The effect of surface charge on in vivo biodistribution of PEG-oligocholic acid based micellar nanoparticles. Biomaterials 2011; 32: 3435-3446.

37. Domański DM, Klajnert B, Bryszewska M. Influence of PAMAM dendrimers on human red blood cells. Bioelectrochemistry 2004; 63: 189-191.

38. Wang $\mathrm{W}$, Xiong $\mathrm{W}$, Zhu $\mathrm{Y}$ et al. Protective effect of PEGylation against poly(amidoamine) dendrimer-induced hemolysis of human red blood cells. J Biomed Mater Res B Appl Biomater 2010; 93: 59-64.

39. Ding $\mathrm{H}$, Yong $\mathrm{K}-\mathrm{T}$, Roy I et al. Bioconjugated PLGA-4-arm-PEG branched polymeric nanoparticles as novel tumor targeting carriers. Nanotechnology 2011; 22: 165101.

40. Jung SH, Jung SH, Seong $\mathrm{H}$ et al. Polyethylene glycol-complexed cationic liposome for enhanced cellular uptake and anticancer activity. Int J Pharm 2009; 382: 254-261.

41. He C, Hu Y, Yin L et al. Effects of particle size and surface charge on cellular uptake and biodistribution of polymeric nanoparticles. Biomaterials 2010; 31: 3657-3666.

42. Roser M, Fischer D, Kissel T. Surface-modified biodegradable albumin nano- and microspheres. II: effect of surface charges on in vitro phagocytosis and biodistribution in rats. Eur J Pharm Biopharm 1998; 46: 255-263.

43. Lundqvist M, Stigler J, Elia G et al. Nanoparticle size and surface properties determine the protein corona with possible implications for biological impacts. Proc Natl Acad Sci U S A 2008; 105: 14265-14270.

44. Hafez IM, Cullis PR. Cholesteryl hemisuccinate exhibits $\mathrm{pH}$ sensitive polymorphic phase behavior. Biochimica et Biophysica Acta (BBA) Biomembranes 2000; 1463: 107-114.

45. Reddy LH, Murthy RS. Pharmacokinetics and biodistribution studies of Doxorubicin loaded poly(butyl cyanoacrylate) nanoparticles synthesized by two different techniques. Biomed Pap Med Fac Univ Palacky Olomouc Czech Repub 2004; 148: 161-166.

46. Osman A-MM, Nemnem MM, Abou-Bakr AA et al. Effect of methimazole treatment on doxorubicin-induced cardiotoxicity in mice. Food and Chemical Toxicology 2009; 47: 2425-2430.

47. Pereverzeva E, Treschalin I, Bodyagin D et al. Influence of the formulation on the tolerance profile of nanoparticle-bound doxorubicin in healthy rats: Focus on cardio- and testicular toxicity. Int J Pharm 2007; 337: 346-356.

48. Pereverzeva E, Treschalin I, Bodyagin D et al. Intravenous tolerance of a nanoparticle-based formulation of doxorubicin in healthy rats. Toxicology Letters 2008; 178: 9-19. 